\title{
Effect of the downregulation of SMYD3 expression by RNAi on RIZ1 expression and proliferation of esophageal squamous cell carcinoma
}

\author{
SHANG-WEN DONG ${ }^{1}$, HAO ZHANG $^{1}$, BAO-LI WANG $^{2}$, PEI SUN $^{2}$, YUAN-GUO WANG ${ }^{1}$ and PENG ZHANG \\ ${ }^{1}$ Department of Cardiothoracic Surgery, Tianjin Medical University General Hospital, Tianjin, Heping 300052; \\ ${ }^{2}$ Tianjin Institute of Endocrinology, Tianjin Medical University, Tianjin, Heping 300070, P.R. China
}

Received March 10, 2014; Accepted May 2, 2014

DOI: $10.3892 /$ or.2014.3307

\begin{abstract}
The present study aimed to investigate the expression and role of SET and MYND domain-containing protein 3 (SMYD3) in esophageal squamous cell carcinoma; to observe the proliferation of esophageal squamous cell carcinoma after suppression of SMYD3 expression; and to explore the effect of SMYD3 downregulation on the expression of retinoblastoma protein-interacting zinc finger gene 1 (RIZ1). Tissues from 11 patients, including cancer and normal esophageal tissues, were obtained by surgery to observe the SMYD3 protein expression immunohistochemistry. Esophageal squamous cell carcinoma TE13 cells were transfected with four different SMYD3-shRNA plasmids, and SMYD3 mRNA expression levels were assessed to select the most efficient interfering plasmid. After SMYD3 downregulation in TE13 cells, mRNA and protein expression levels of SMYD3 and RIZ1 were determined using RT-PCR and western blotting, and cell proliferation was evaluated by the MTT method. In all 11 tissue paired samples, SMYD3 protein expression was higher in the cancer tissues $(72.7 \% ; 8 / 11)$, than that in the normal tissues $(18.2 \% ; 2 / 11)$ (Fisher's exact test, $\mathrm{P}=0.03)$. The mRNA expression levels of SMYD3 were significantly decreased by RNA interference $(\mathrm{P}<0.05)$, and plasmid SMYD3-shRNA-1242 was determined to be the most effective. Compared with the controls, transfection with the SMYD3-shRNA interfering plasmid significantly reduced the SMYD3 mRNA and protein expression levels in TE13 cells $(\mathrm{P}<0.05)$, whereas the expression levels of the anti-oncogene RIZ1 were increased $(\mathrm{P}<0.05)$. The MTT assay showed that ablation of SMYD3
\end{abstract}

Correspondence to: Professor Peng Zhang or Dr Shang-Wen Dong, Department of Cardiothoracic Surgery, Tianjin Medical University General Hospital, Tianjin Medical University, No. 154 Anshan Road, Tianjin, Heping 300070, P.R. China

E-mail: zhang_peng6036@yeah.net

E-mail: dongshangwen@yahoo.com

Key words: SMYD3, RIZ1, esophageal squamous cell carcinoma, RNA interference expression significantly inhibited proliferation of TE13 cells $(\mathrm{P}<0.05)$. SMYD3 may participate in the biological activity of esophageal squamous cell carcinoma, as overexpression of SMYD3 correlates with its occurrence and its downregulation inhibits cancer cell proliferation. The shRNA efficiently downregulated SMYD3 in TE13 cells, which represents an SMYD3-interfered cell-test-model for future experiments. RNAi suppression of SMYD3 promoted the expression of RIZ1 in TE13 cells, suggesting a signal transduction pathway between SMYD3 and RIZ1. The SMYD3-RIZ1 pathway may represent a therapeutic target for esophageal squamous cell carcinoma.

\section{Introduction}

Esophageal cancer is one of the most common human malignant tumors in the digestive system; more than 200,000 individuals die from esophageal cancer each year worldwide, while more than 150,000 individuals die of esophageal cancer each year in China (1). Thus, esophageal cancer is a serious threat to human health. Current methods of early diagnosis and treatment are not satisfactory. The most common pathological type of esophageal cancer is squamous cell carcinoma (ESCC), particularly in China. In recent years, with the continuous development of molecular biology, genetics, epigenetics, immunology and histochemistry, scientists have gradually started to reveal the genetic and molecular processes underlying the development of esophageal cancer and their clinical application.

The important role of histone methylation modification and its relationship with DNA methylation have received much attention from researchers. SET and MYND domain-containing protein 3 (SMYD3) is a type of histone methyltransferase. Recent studies have shown that SMYD3 is extensively expressed in human hepatocellular carcinoma, colon, breast, cervical cancer and other malignant tumors, where it is involved in proliferation, apoptosis, invasion and metastasis. However, the expression of SMYD3 in ESCC has not been reported. The relatively recent technology of RNA interference (RNAi) has been widely used in research related to gene expression in tumor cells, and is expected to be developed as a new tumor therapy. If SMYD3 expression is associated with ESCC, such as in tumor cell proliferation, 
Table I. SMYD3 interference target sequence.

\begin{tabular}{llccc}
\hline Clone name & Symbol & Location & Length & Target sequences \\
\hline HSH017124-1-CH1(OS238397) & SMYD3 & 290 & 19 & gatggagcaccttcagaat \\
HSH017124-2-CH1(OS238398) & SMYD3 & 658 & 19 & catctgctacctggatatg \\
HSH017124-3-CH1(OS238399) & SMYD3 & 714 & 19 & accagtactgctttgaatg \\
HSH017124-4-CH1(OS238400) & SMYD3 & 763 & 19 & ggatgctgatatgctaact \\
\hline
\end{tabular}

SMYD3, SET and MYND domain-containing protein 3.

invasion or other malignant cell biologic behaviors, then SMYD3 would offer a new diagnostic and therapeutic target for ESCC. Its downregulation by RNAi would represent a novel ESCC therapy. The present study aimed to evaluate the biological role of SMYD3 in ESCC and to clarify the relationship between SMYD3 and the important anti-oncogene retinoblastoma protein-interacting zinc finger gene 1 (RIZ1) in ESCC.

\section{Materials and methods}

Ethics statement. The research was conducted in accordance with the Declaration of Helsinki. All experimental protocols were approved by the Ethics Committee for the Use of Human Subjects of Tianjin Medical University General Hospital. Patients provided written consent to participate in this study, and this consent was also approved by the Ethics Committee for the Use of Human Subjects of Tianjin Medical University General Hospital.

Cell culture and tissues. Human ESCC cell line TE13 was purchased from ATCC (Rockville, MD, USA) and cultured in RPMI-1640 (HEPES $4.76 \mathrm{~g} ; \mathrm{NaCO}_{3} 2.0 \mathrm{~g}$; RPMI-1640 $10.4 \mathrm{~g} ; \mathrm{ddH}_{2} \mathrm{O} 1,000 \mathrm{ml}$ ) media supplemented with $10 \%$ fetal calf serum, $2 \mathrm{mM} 1 \mathrm{X}$ L-glutamine, $100 \mathrm{U} / \mathrm{ml}$ penicillin and $100 \mu \mathrm{g} / \mathrm{ml}$ streptomycin (Life Technologies, Carlsbad, CA, USA). Cells were maintained at $37^{\circ} \mathrm{C}$ in a humidified atmosphere with $5 \% \mathrm{CO}_{2}$.

Carcinoma and distal ending normal tissues $(>5 \mathrm{~cm}$ from the tumor) were obtained in our department during surgical excision from 11 patients with ESCC. All specimens were placed in liquid nitrogen immediately after resection and stored at $-80^{\circ} \mathrm{C}$ until RNA or genomic DNA (gDNA) extraction. No patient had received chemotherapy or radiation therapy before surgery. All patients were confirmed to have ESCC by pathological tests.

Immunohistochemistry. Formalin-fixed, paraffin-embedded sections of ESCC and distal ending normal tissues were subjected to immunostaining with a rabbit polyclonal antibody against SMYD3. Briefly, 5- $\mu \mathrm{m}$ thick tissue sections were deparaffinized, rehydrated and subjected to antigen retrieval by boiling in sodium citrate buffer $(10 \mathrm{mM}, \mathrm{pH}$ 6.0). The sections were incubated at $4^{\circ} \mathrm{C}$ overnight with SMYD3 antibody AP1202c (1:75 dilution; Abgent, San Diego, CA, USA) and then stained with 3,3'-diaminobenzidine. After visualization of immunoreactivity, the sections were counterstained with hematoxylin and mounted. Adjacent non-cancerous esophageal tissues were used as internal positive controls.

The stains were graded as follows: i) positive when immunoreactivity was equivalent to that observed in normal esophageal squamous cells or had moderately decreased; and ii) negative when immunoreactivity was weak or absent. Under a high-resolution optical microscope (SP200), four different fields of view were selected randomly, and the percentage of positive cells among the total cells was calculated. If the positive cell fraction was $<10 \%$, it was scored as 1 ; if the fraction was $>10 \%$ but $\leq 50 \%$, it was scored as 2 ; if the fraction was $>50 \%$ but $\leq 75 \%$, it was scored as 3 ; and if the fraction was $>75 \%$, it was scored as 4 . Different degrees of pigmentation were also scored: negative as 1 ; weak pigmentation as 2 ; moderate pigmentation as 3; and strong pigmentation as 4 . By combining the above two indices, the final results became: $\leq 4$ as $(-),>4$ but $\leq 8$ as $(+),>8$ but $\leq 12$ as $(++),>12$ but $\leq 16$ as $(+++)$ and $>16$ as $(++++)$.

Design and construction of the expression plasmid of SMYD3-shRNA; plasmid transformation, extraction, identification and transfection of cells. For the knockdown of SMYD3 expression, according to the design principles of shRNA, specific target sequences from the SMYD3 mRNA (NM_022743.1) coding sequence were identified (Table I). Target oligonucleotide sequences were designed and synthesized into eukaryotic expression plasmids named 1241-1244. The Guangzhou Synthetic Genes Company synthesized the above materials.

Briefly, 2x10 5 TE13 cells/well in 6-well plates were transfected with $4 \mu \mathrm{g}$ of specific or control shRNA using Lipofectamine 2000 ${ }^{\mathrm{TM}}$ (Life Technologies). After incubation for the designated time intervals, the cells were prepared for real-time PCR and western blotting.

RNA extraction and reverse transcription reaction. Total RNA from cells and tissues was isolated using the TRIzol reagent (Life Technologies), according to the manufacturer's recommendations. Cellular RNA was isolated from $5 \times 10^{6}$ to $1 \times 10^{7}$ cells by $1 \mathrm{ml}$ TRIzol decomposition, and tissues samples were ground into a fine powder using a mortar and pestle, and then incubated in TRIzol solution (100 g/l) for $15 \mathrm{~min}$. One fifth of the volume of chloroform was then added. After vigorous agitation and standing for $5 \mathrm{~min}$, the inorganic phase was separated by centrifugation at $12,000 \mathrm{x} \mathrm{g}$ for $15 \mathrm{~min}$ at $4^{\circ} \mathrm{C}$. RNA was then precipitated in the presence of an equal volume of isopropanol and centrifuged at $12,000 \mathrm{x} \mathrm{g}$ for $10 \mathrm{~min}$ 
Table II. SMYD3 protein expression in ESCC and adjacent non-cancerous tissues by immunohistochemical detection.

\begin{tabular}{lcccccccc}
\hline & \multicolumn{7}{c}{ Positive } & \\
\cline { 2 - 6 } Group & ++++ & +++ & ++ & + & Total & Negative & Total samples & Positive rate (\%) \\
\hline Cancer & 0 & 2 & 4 & 2 & 8 & 3 & 11 & 72.7 \\
Adjacent non-cancerous & 0 & 0 & 0 & 2 & 2 & 9 & 11 & 18.2 \\
F-value & 6.3 & & & & & & & \\
P-value & 0.03 & & & & & & \\
\hline
\end{tabular}

SMYD3, SET and MYND domain-containing protein 3; ESCC, esophageal squamous cell carcinoma.

at $4^{\circ} \mathrm{C}$. RNA pellets were washed with $1 \mathrm{ml} 75 \%$ ice-cold ethanol [diethylpyrocarbonate (DEPC)-treated], centrifuged at $8,000 \mathrm{x}$ g for $5 \mathrm{~min}$ at $4^{\circ} \mathrm{C}$ and then dissolved in DEPC-treated $\mathrm{H}_{2} \mathrm{O}$. A UV spectrophotometer (Beckman Coulter, Brea, CA, USA; absorbance at 260 and $280 \mathrm{~nm}$ ) and $1.2 \%$ denaturing agarose gels were used to determine the concentration and quality of the total RNA. For quantitative real-time PCR analysis, $2 \mu \mathrm{g}$ of RNA was reverse transcribed using reverse transcriptase M-MLV, ribonuclease inhibitor and dNTP mixture (all from Takara, Japan), according to the manufacturer's protocol. The cDNA templates were then subjected to PCR amplification.

$R T$-PCR. Two microliters of cDNA from the TE13 cell lines was used as the template to amplify specific fragments in a $25 \mu 1$ reaction mixture, under the following conditions: denaturation at $94^{\circ} \mathrm{C}$ for $3 \mathrm{~min} ; 30$ cycles at $94^{\circ} \mathrm{C}$ for $30 \mathrm{sec}$, at $55^{\circ} \mathrm{C}$ for $30 \mathrm{sec}$, at $72^{\circ} \mathrm{C}$ for $60 \mathrm{sec}$; and then extension at $72^{\circ} \mathrm{C}$ for $10 \mathrm{~min}$. As a control for cDNA integrity, GAPDH expression was also analyzed. The primer $(10 \mu \mathrm{M})$ sets were: SMYD3 forward, 5'-AACGGCTTCCCGATATCA-3' and reverse, 5'-ATCACTTGAACCCCTCTGA-3'; GAPDH forward, 5'-GA AGGTGAAGGTCGGAGTC-3' and reverse, 5'-GGGTGGAA TCATATTGGAAC-3'. The SMYD3 primer set yielded a band of $169 \mathrm{bp}$ and the GAPDH primer set yielded a band of $152 \mathrm{bp}$. Five microliters of the RT-PCR reaction product was analyzed by electrophoresis on a $1 \%$ agarose gel, and the electrophoresis images were scanned using a UV spectrophotometer (Beckman Coulter).

Western blotting. Tissues from each group were homogenized in RIPA buffer $(50 \mathrm{mmol} / 1$ Tris- $\mathrm{HCl}, \mathrm{pH} 7.4 ; 150 \mathrm{mmol} / 1$ $\mathrm{NaCl} ; 1 \%$ Nonidet $\mathrm{P}-40 ; 0.5 \%$ sodium deoxycholate; $0.1 \%$ SDS; $1 \mathrm{mmol} / 1$ EDTA; $1 \mathrm{mmol} / 1 \mathrm{PMSF} ; 1 \mathrm{mg} / \mathrm{ml}$ aprotinin). With 2 min fully cracking the cell then $14,000 \mathrm{x}$ g centrifugation for $5 \mathrm{~min}$, the supernatant was collected and the protein concentrations were determined using a bicinchoninic acid (BCA) protein assay kit (Pierce, Rockford, IL, USA). Subsequently, $30 \mu \mathrm{g}$ of whole-cell lysate was separated on an $8 \%$ SDS-PAGE gel, transferred to nitrocellulose (NC) membranes (Amersham Biosciences, Piscataway, NJ, USA) and immunoblotted with the following antibodies: anti- $\beta$ actin, control; primary antibodies, 1:2,000 dilution; secondary antibodies goat anti-mouse, 1:5,000 dilution (all from Abcam, UK). A PowerLook scanner (UMAX, Taiwan) was used to analyze the films and ImageQuant software (GE, USA) quantified the band intensities. The control samples (TE13 cells; TE-13 cells transfected with the blank plasmid) were treated in the same manner. The relative expression of SMYD3 = the grey value of SMYD3 protein/grey value of $\beta$-actin.

3-(4,5-Dimethylthiazol-2-yl)-2,5-diphenyltetrazolium bromide (MTT) assay. Some TE13 cells, which are in the logarithmicincrease period, were selected and inoculated on 96-well plates with each well having $0.8 \times 10^{4} / \mathrm{ml}(200 \mu \mathrm{l})$. The original culture medium was discarded and the cells were transfected when the cell density reached approximately $70-80 \%$ and divided into groups (blank, negative control and SMYD3-shRNA) as described above. Each group was assigned 8 wells as a parallel experiment. After $48 \mathrm{~h}$, one of the 96-well plates was examined according to following steps: adding $20 \mu \mathrm{l}$ MTT solution $(5 \mathrm{~g} / \mathrm{l})$, removing the upper clear part after $5 \mathrm{~h}$ at $37^{\circ} \mathrm{C}$, adding $150 \mu \mathrm{l}$ dimethylsulfoxide (Newprobe, Beijing, China) and vibrating at low speed for $10 \mathrm{~min}$ to thoroughly dissolve the crystals, measuring ray density at a wavelength of $492 \mathrm{~nm}$ using a microplate reader, and using the blank cells as zero for the calculation of the restraining rates of cell proliferation. Inhibition ratio of cell proliferation $=[($ comparison group A492 - blank group A492 $)-$ (experiment group A492 - blank group A492)]/(comparison group A492-experiment group A492) x 100\%.

Statistical methods. SPSS 19.0 statistical software was used for statistical analysis. Fisher's exact test was used to analyze the positive rate between two groups. All values are expressed as the means \pm standard deviation (SD). For more than one group, the mean was compared using one-way ANOVA analysis of variance. Means of more than two groups were compared using the least squares difference (LSD) method. Differences with a P-value $<0.05$ were considered to indicate a statistically significant result.

\section{Results}

SMYD3 is significantly expressed in ESCC tissues. Fig. 1 shows the immunohistochemical staining for SMYD3 using a rabbit anti-SMYD3 antibody. In the ESCC tissue samples (Fig. 1A and C), SMYD3 staining was concentrated in the submucosa, mainly in the esophageal tissue cell cytoplasm. In the normal esophageal tissues, even at x200 magnification, little or no SMYD3 staining was visible. A semi-quantitative 
A

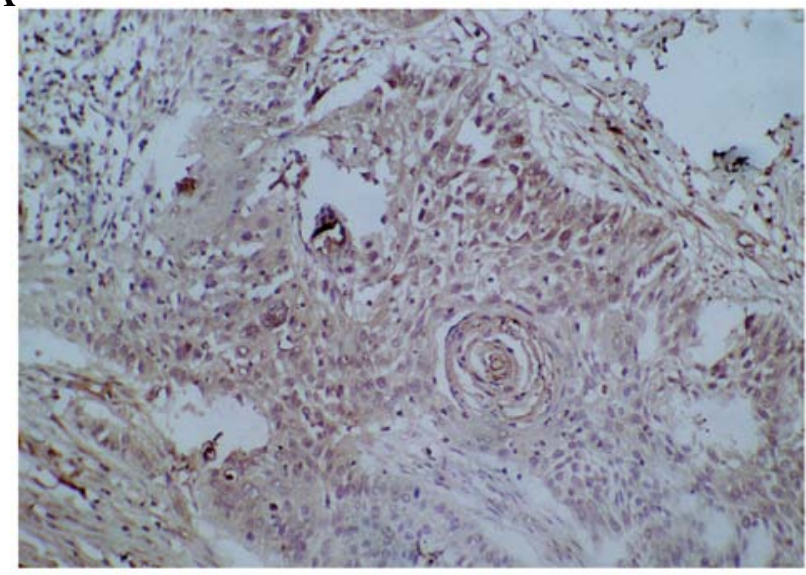

C

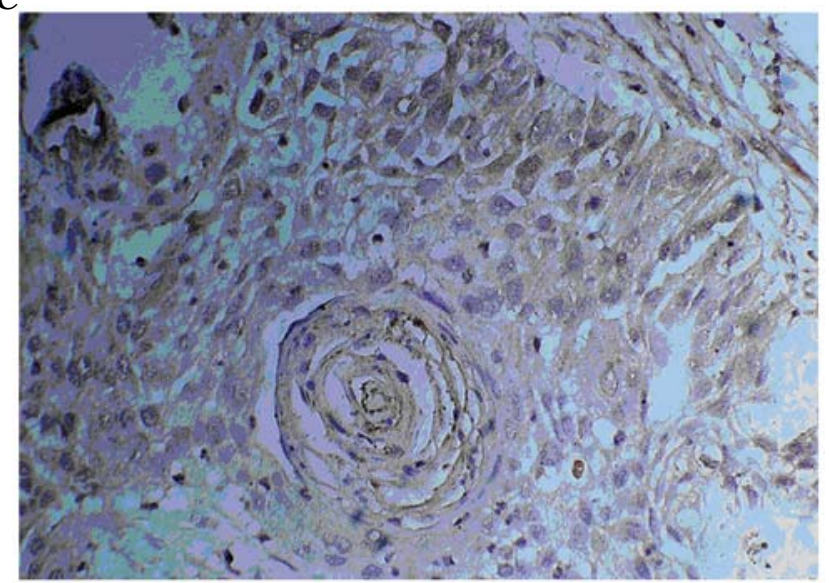

B

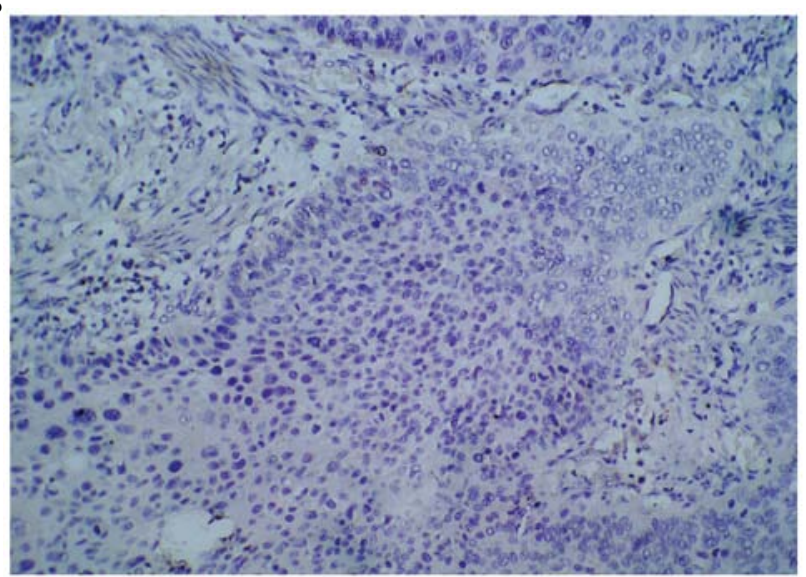

D

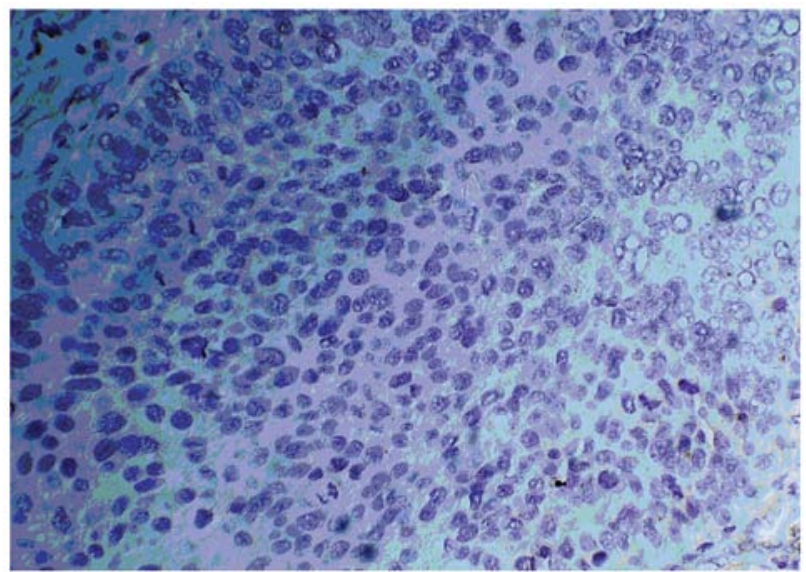

Figure 1. Immunohistochemical detection of SMYD3 in ESCC and normal tissue samples. (A) SMYD3 protein expression in an ESCC esophageal biopsy (SPx 100); (B) esophageal SMYD3 protein expression in a normal tissue slice (SPx100); (C) SMYD3 protein expression in an ESCC esophageal biopsy (SPx200); (D) esophageal SMYD3 protein expression in a normal tissue slice (SPx200). SMYD3, SET and MYND domain-containing protein 3; ESCC, esophageal squamous cell carcinoma.

A

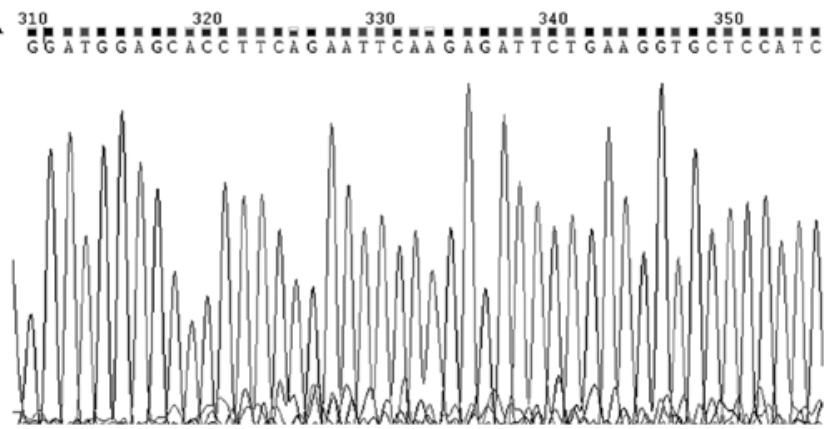

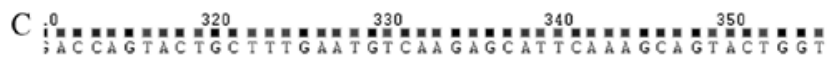

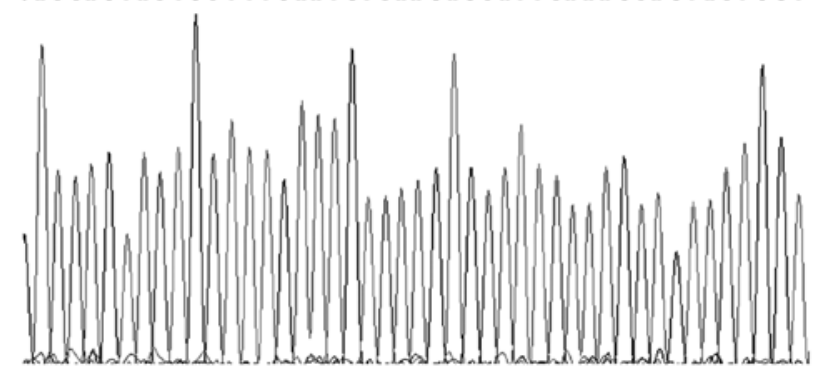

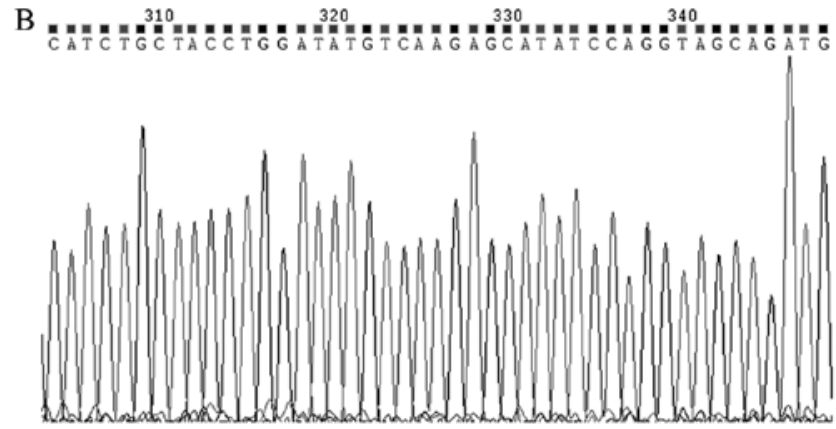

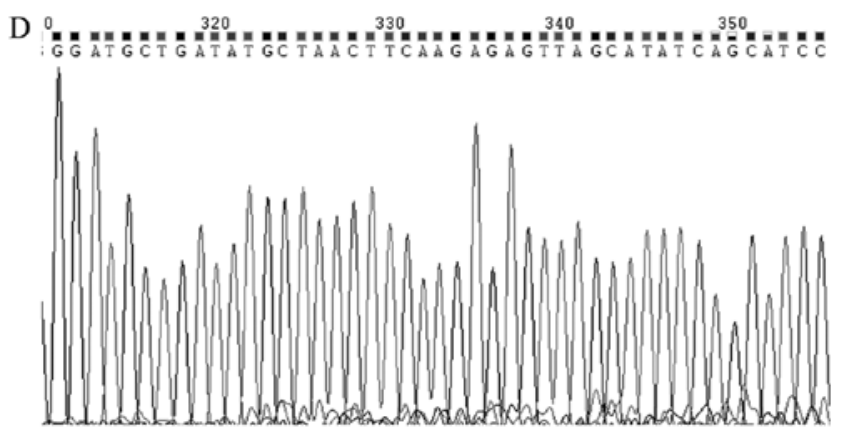

Figure 2. (A-D) Sequence chromatograms of the shRNA sequences contained in plasmids P1241-P1244. 


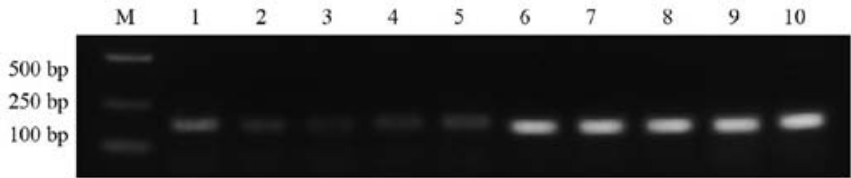

Figure 3. RNA interference of SMYD3 mRNA expression. Lane M, DNA markers, from top to bottom 500, 250 and $100 \mathrm{bp}$; lane 1, SMYD3-negative control; lanes 2-5, SMYD3 mRNA expression in cells transfected with shRNA plasmids P1241-P1244; lanes 6-10, GAPDH mRNA expression in the control, and in the cells transfected with plasmids P1241-P1244. SMYD3, SET and MYND domain-containing protein 3.
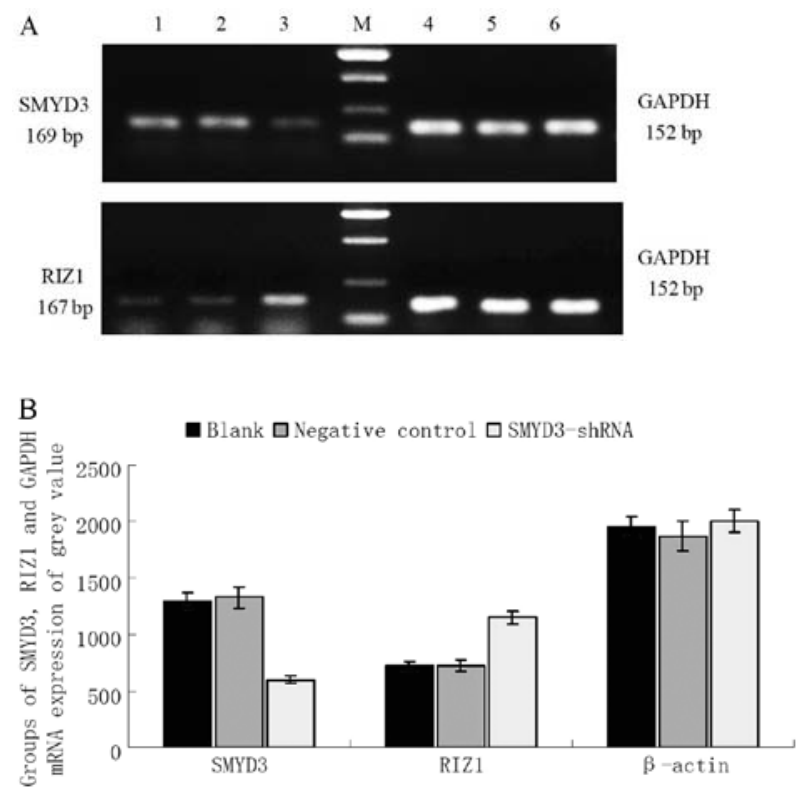

Figure 4. RNA interference of SMYD3 increases the expression of RIZ1 mRNA. (A) Upper panel: lanes 1 and 2, SMYD3 expression in the blank and negative controls; lane 3, SMYD3 expression following treatment with SMYD3-shRNA; M, DNA markers, from top to bottom 750, 500, 200 and $100 \mathrm{bp}$; lanes 4-6, GAPDH mRNA expression in the cells of lanes 1-3. Lower panel: lanes 1 and 2, RIZ1 expression in the blank and negative controls; lane 3, RIZ1 expression following treatment with SMYD3-shRNA; M, DNA markers, from top to bottom 750, 500, 200 and $100 \mathrm{bp}$; lanes 4-6, GAPDH mRNA expression in the cells of lanes 1-3. (B) Graph generated from the RT-PCR analyses in A. SMYD3, SET and MYND domain-containing protein 3; RIZ1, retinoblastoma protein-interacting zinc finger gene 1 .

method was used to count the SMYD3-positive cells to calculate the number of positive cells between the two groups. The results are shown in Table II. Histopathological biopsies from 11 cases of esophageal cancer showed an SMYD3-positive expression rate of $72.7 \%(8 / 11)$. In the normal esophageal tissues, the SMYD3-positive rate was 18.2\% (2/11) (Fisher's exact test, $\mathrm{P}=0.03$; Mann-Whitney $\mathrm{U}$ rank sum test, $\mathrm{P}<0.05$ ).

Testing shRNA constructs for their ability to suppress SMYD3 $m R N A$ expression. Four shRNA plasmids were constructed and tested for their ability to suppress SMYD3 mRNA expression. The sequences of the four shRNAs in plasmids SMYD3-shRNA-1241 - SMYD3-1244 are shown in Fig. 2. The four plasmids were transfected into TE13 ESCC cells, and RT-PCR was used to determine the quantities of SMYD3 mRNA following treatment with each shRNA. Fig. 3 shows that all four shRNAs significantly reduced the expression of
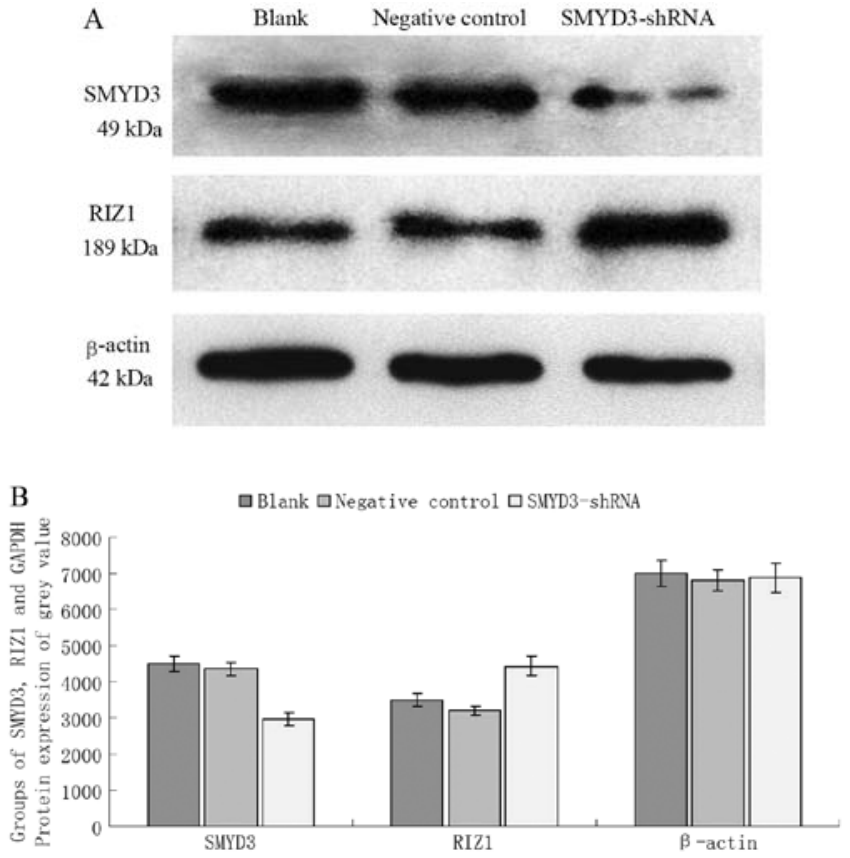

Figure 5. RNA interference of SMYD3 increases the expression of RIZ1 protein. (A) Western blotting showing SMYD3, RIZ1 and $\beta$-actin protein expression in the blank and negative controls, and in the cells treated with the SMYD3-shRNA. (B) Graph generated from the scanned band intensities from the western blot analyses in A. SMYD3, SET and MYND domain-containing protein 3; RIZ1, retinoblastoma protein-interacting zinc finger gene 1.

SMYD3 mRNA relative to the blank control $(\mathrm{P}<0.05)$. For constructs 1241-1244, the inhibition rates were 29.9, 52.2, 38.4 and $38.3 \%$, respectively. The performance of construct 1242 was the best $(52.2 \%)$ and the differences were statistically significant between 1242 and the other groups $(\mathrm{P}<0.05)$. Among constructs 1241, 1243 and 1244, there was no statistically significant difference in their performances $(\mathrm{P}>0.05)$. The expression of GAPDH mRNA was not significantly different between the groups $(\mathrm{P}>0.05)$. Therefore, construct 1242 was used for subsequent experiments.

Effect of suppression of SMYD3 on the expression of RIZ1 $m R N A$. TE13 cells transfected with shRNA construct 1242 were subjected to RT-PCR to determine the levels of SMYD3, RIZ1 and GAPDH (control) (Fig. 4). The expression of SMYD3 was significantly reduced relative to the blank group and the negative control group $(\mathrm{P}<0.05)$. SMYD3 expression was not significantly different between the negative control group and the blank group ( $\mathrm{P}>0.05$ ) (Fig. 4A and B). RIZ1 mRNA expression was significantly increased in the cells transfected with SMYD3-shRNA-1242, relative to the blank group and negative control group $(\mathrm{P}<0.05)$ (Fig. 4A and $\mathrm{B})$. RIZ1 expression in the negative control group and the blank group was not significantly different $(\mathrm{P}>0.05)$. Among all three groups, the expression of GAPDH mRNA was not significantly different $(\mathrm{P}>0.05)$.

Effect of suppression of SMYD3 on the expression of RIZ1 protein. Western blotting of TE13 cells transfected with shRNA construct 1242 was used to determine the effect of suppressing SMYD3 expression on the levels of the RIZ1 


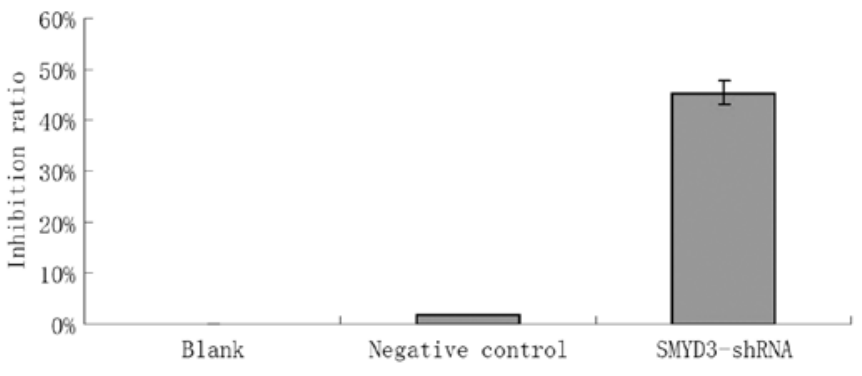

Figure 6. Inhibition of cell proliferation as measured by the MTT assay. The graph shows the percentage of inhibition of proliferation in the blank and negative controls and in cells treated with SMYD3-shRNA. SMYD3, SET and MYND domain-containing protein 3.

protein. Fig. 5A shows that compared with the blank control and negative control, the levels of the SMYD3 protein were obviously reduced in the shRNA-treated cells. The levels of RIZ1 were obviously increased in the shRNA-treated cells. The levels of $\beta$-actin appeared similar across all samples. The band intensities were scanned and quantified to permit a statistical analysis of the protein expression data (Fig. 5B). The difference between the SMYD3 protein expression in the experimental group was significant compared with both controls $(\mathrm{P}<0.05)$. The controls were not significantly different to each other $(\mathrm{P}>0.05)$. The difference between the RIZ1 protein expression in the experimental group was significant compared with both controls $(\mathrm{P}<0.05)$. The controls were not significantly different to each other $(\mathrm{P}>0.05)$. $\beta$-actin expression in all three groups was not significantly different $(\mathrm{P}>0.05)$.

Effect of suppression of SMYD3 expression on the proliferation of TE13 ESCC cells. TE13 cells transfected with shRNA-1242, blank control cells and negative control cells were cultured for $48 \mathrm{~h}$ in vitro and then subjected to the MTT test to assay the inhibition of their proliferative ability. Cells transfected with the shRNA showed an inhibition of their proliferative ability of $45.41 \%$, whereas the negative control's inhibition rate was $1.86 \%(\mathrm{P}<0.05)$. Compared with the blank group, the negative control group showed no significant inhibition of proliferation $(\mathrm{P}>0.05)$ (Fig. 6).

\section{Discussion}

In 2004, Hamamoto et al (2) and Sims and Reinberg (3) were the first to identify the histone methyltransferase SET and MYND domain containing 3 (SMYD3) in human hepatocellular carcinoma and colon cancer cells using a cDNA chip and semi-quantitative RT-PCR. SMYD3 was found to methylate chromosomal histone $\mathrm{H} 3 \mathrm{~K} 4$ twice (dimethylated form) or three times (trimethylated form), which altered the expression of 80 candidate genes, of which 61 were upregulated and 19 were downregulated. Among these genes are cancer-associated genes, such as tumor-suppressor genes, signal transduction genes and cell cycle regulation genes, which can promote cell adhesion, proliferation, invasion and inhibit tumor cell apoptosis. The SMYD3 gene is located on human chromosome 1q44 and comprises 12 exons. The SMYD3 mRNA (GenBank login number: NM_022743) comprises 1,477 bp, encoding a 369 -amino acid protein. The SMYD3 protein contains two important domains. Firstly, the myeloid translocation protein 8 , Nervy, DEAF1 (MYND) zinc finger domain, located at amino acid 1-28 at the N-terminus, can specifically combine with gene sequences 5'-CCCTCC-3' or 5'-GGAGGG-3' in promoter regions, promoting the SET domain methylation function and influencing gene transcription. Secondly, at position $89-180$ is the $\mathrm{Su}(\mathrm{var}) 3-9$, enhancer-of-zeste trithorax (SET) lysine methyltransferase domain, comprising the methylation transferase function, which can specifically methylate chromosomal histone $\mathrm{H} 3 \mathrm{~K} 4$, which loosens and opens the spatial structure of the chromosome (4-7).

In the present study, we used immunohistochemistry to detect SMYD3 protein expression in ESCC and corresponding normal esophageal tissues and showed that SMYD3 expression was significantly higher in the ESCC group than in the corresponding normal esophageal tissues. The results suggest that high expression of SMYD3 is related to the occurrence of ESCC. Although further study of the role and mechanism of SMYD3 in the development of ESCC is required, it is likely to lead to new methods of early diagnosis of esophageal cancer and to the development of new antitumor drugs or gene therapy. To investigate the effect of suppressing SMYD3 expression, we produced and tested an RNA interference plasmid SMYD3shRNA-1242 in TE13 cells. Using semi-quantitative RT-PCR and western blotting, we observed that the levels of SMYD3 mRNA and protein in the interference group were lower than these levels in the negative control and blank control groups. The two control groups showed no significant differences, and the expression level of the $\beta$-actin internal reference was similar among all three groups, Thus, RNAi technology could successfully and efficiently reduce SMYD3 gene expression in ESCC cells, representing an SMYD3 downregulation model for subsequent research. Furthermore, the MTT test showed that the cell proliferation in the experimental group was significantly reduced $(\mathrm{P}<0.001)$ compared with the negative control and blank control groups. This result strongly suggests that the SMYD3 gene is a key link in the process of cell proliferation in ESCC, where it likely exerts its effect through its transcriptional regulatory activity, directly or indirectly changing the expression of proto-oncogenes (e.g., c-met, c-jun and Wnt10B), signal transduction-related genes (e.g., GnRF2 and RAB40C) or cell cycle regulatory genes (e.g., CDK2 and DNA topoisomerase II- $\beta$ ). However, the specific molecular mechanism and signal transduction pathway molecules remain to be determined $(3,8)$.

In addition, we tested retinoblastoma protein-interacting zinc finger gene 1 (RIZ1) expression after SMYD3 downregulation. Oncogene activation and tumor-suppressor gene inactivation are important mechanisms of tumorigenesis and progression. RIZ1 belongs to the nucleoprotein methyltransferase superfamily (9), which was first reported by Buyse et al, who identified a retinal cell tumor-suppressor protein by functional screening (9). RIZl methylates histones through its PR structure domain on H3K9, leading to the inhibition of gene transcription. Unlike RIZ1, RIZ2 starts transcription from exon 6, thereby lacking the first five exons, which encode the tumor-suppressor PR domain. Imbalances between RIZ1 and RIZ2 are an underlying cause of tumorigenesis. RIZl is widely expressed in multiple tissues and cells; however, its expression is low or absent in tumor tissue, both 
in vivo and in vitro, which correlates with its tumor inhibitory function $(10,11)$. Further research confirmed that the expression of RIZl is significantly related to tumorigenesis and progression in malignant tumors. Our laboratory has reported many research results for tumor-suppressor gene RIZ1 in ESCC, further confirming that RIZ1 is an important inhibitory factor in ESCC (12-15). In the present study, we showed that the mRNA and protein expression of RIZ1 both increased in the SMYD-shRNA group compared with the negative control and blank groups. There were no significant differences between the negative control and blank group or between the internal reference $\beta$-actin in all three groups. Thus, suppression of SMYD3 expression could increase RIZ1 expression in ESCC cells. This suggests the existence of a signaling pathway between SMYD3 and RIZ1, where alterations in the expression of the SMYD3 gene could affect tumor cell biology.

As a histone methylation transferase, the substrate of SMYD3 is mainly histone. Although the SET domain of SMYD3 is the structural domain responsible for the histone methyltransferase function, SMYD3 also possess a zf-MYND zinc finger structural domain. The downregulation of the expression of RIZ1 may involve the zf-MYND zinc finger structure of SMYD3. Zinc finger structure domains are important transcription elements that can combine with the specific binding elements (SBEs) of the target DNA promoter. The presence of the zf-MYND in SMYD3 suggests that SMYD3 could not only regulate gene expression via histone methylation by the SET domain, but also directly through the zinc finger domain. Related research has identified the SBEs for zf-MYND as 5'-CCCTCC-3' and 5'-GGAGGG-3' (5). Notably, SBE 5'-GGAGGG-3' is present in the RIZ1 promoter sequence (16). Thus, SMYD3 could directly regulate RIZ1 expression by the binding of its zf-MYND zinc finger to the RIZ1 SBE sequence. Of course, it is possible that SMYD3 indirectly regulates the RIZ1 gene by binding to an SBE in another DNA sequence. For example, SMYD3 binding to the SBE of DNA methyltransferase (DNMT) could affect the RIZ1 promoter methylation levels, which would regulate the expression of RIZ1. The exact mechanism of the regulation of RIZ1 expression by SMYD3 and the effect of the proposed SMYD3-RIZ1 signaling pathway in ESCC remain undetermined. We hypothesize that the mechanism is closely related to epigenetics. We will perform further studies in this area.

\section{Acknowledgements}

This study was supported by grants from the National Natural Science Foundation of China (no. 81201945), the Science Foundation of Tianjin Medical University (no. 2011KY08), the Doctoral Program of Higher Education Research Fund (no. 20121202110009), and the Natural Science Foundation of Tianjin (no. 10JCYBJC11300).

\section{References}

1. Dong SW, Ma L, Xu N, Yan HQ, Liu HY, Li YW and Zhang P: Research on the reactivation of Syk expression caused by the inhibition of DNA promoter methylation in the lung cancer. Neoplasma 58: 89-95, 2011.

2. Hamamoto R, Fumkawa Y, Morita M, et al: SMYD3 encodes a histone methyltransferase involved in the proliferation of cancer cells. Nat Cell Biol 6: 731-740, 2004.

3. Sims RJ III and Reinberg D: From chromatin to cancer: a new histone lysine methyltransferase enters the mix. Nat Cell Biol 6: 685-687, 2004.

4. Sliva FP, Hamamoto R, Kunizaki M, et al: Enhanced methyltransferase activity of SMYD3 by the cleavage of its N-terminal region in human cancer cells. Oncogene 27: 2686-2692, 2008.

5. Chen LB, Xu JY, Yang Z and Wang GB: Silencing SMYD3 in hepatoma demethylates RIZI promoter induces apoptosis and inhibits cell proliferation and migration. World J Gastroenterol 13: 5718-5724, 2007.

6. Kunizaki M, Hamamoto R, Silva FP, Yamaguchi K, Nagayasu T, Shibuya M, Nakamura Y and Furukawa Y: The lysine 831 of vascular endothelial growth factor receptor 1 is a novel target of methylation by SMYD3. Cancer Res 67: 10759-10765, 2007.

7. Liu C, Fang X, Ge Z, et al: The telomerase reverse transcriptase ( $h T E R T$ ) gene is a direct target of the histone methyltransferase SMYD3. Cancer Res 67: 2626-2631, 2007.

8. Luo XG, Xi T, Guo S, et al: Effects of SMYD3 overexpression on transformation, serum dependence, and apoptosis sensitivity in NIH3T3 cells. IUBMB Life 61: 679-684, 2009

9. Buyse IM, Shao G and Huang S: The retinoblastoma protein binds to RIZ, a zinc-finger protein that shares an epitope with the adenovirus E1A protein. Proc Natl Acad Sci USA 92: 4467-4471, 1995.

10. Steele-Perkins G, Fang W, Yang XH, et al: Tumor formation and inactivation of RIZ1, an Rb-binding member of a nuclear proteinmethyltransferase superfamily. Genes Dev 15: 2250-2262, 2001.

11. Robert MF, Morin S, Beaulieu N, Gauthier F, Chute IC, Barsalou A and MacLeod AR: DNMT1 is required to maintain CpG methylation and aberrant gene silencing in human cancer cells. Nat Genet 33: 61-65, 2003.

12. Dong SW, Cui YT, Zhong RR, Liang DC, Liu YM, Wang YG, He Z, Wang S, Liang SJ and Zhang P: Decreased expression of retinoblastoma protein-interacting zinc-finger gene 1 in human esophageal squamous cell cancer by DNA methylation. Clin Lab 58: 41-51, 2012.

13. Dong SW, Zhang P, Liu YM, et al: 2012 Study on RIZ1 gene promoter methylation status in human esophageal squamous cell carcinoma. World J Gastroenterol 18: 576-582, 2012.

14. Dong SW, Li D, Xu C, Sun P, Wang YG and Zhang P: Alteration in gene expression profile and oncogenicity of esophageal squamous cell carcinoma by RIZ1 upregulation. World J Gastroenterol 19: 6170-6177, 2013.

15. Dong S, Zhang P, Liang S, Wang S, Sun P and Wang Y: The role of the retinoblastoma protein-interacting zinc finger gene 1 tumor suppressor gene in human esophageal squamous cell carcinoma cells. Oncol Lett 6: 1656-1662, 2013.

16. Sasaki O, Meguro K, Tohmiya Y, et al: Altered expression or retinoblastoma protein-interacting zinc finger, RIZ, in human leukemia. Br J Haematol 119: 940-948, 2002. 